\author{
L. Guasti • C. Simoni • C. Scamoni • S. Sarzi Braga • C. Crespi • M. Cimpanelli • A.M. Grandi \\ R. Pedretti • L.T. Mainardi • G. Tomei • A. Venco
}

\title{
An unusual case presenting with hypertensive crisis
}

Published online: 31 March 2007

\section{Presentation and history}

Prof. Achille Venco, Prof. Luigina Guasti: A 67-year-old woman was seen at our hospital for an outpatient evaluation. She had a recent history of hypertensive crises (mean blood pressure peaks of 230/120 $\mathrm{mmHg}$ ). She also complained of several symptoms associated with the hypertensive spells, such as fatigue, malaise, irritability, profuse sweating, tremulousness, dyspnoea and abdominal pain, all of them lasting about one hour and with a spontaneous recovery.

She was married, and had no medical history. She did not smoke or consume alcohol. She was not taking any medication and had no allergies.

On examination, blood pressure and heart rate were normal (120/70 $\mathrm{mmHg} ; 64$ beats/min), as were the other vital

L. Guasti $(\varangle)$ • C. Simoni • C. Crespi • M. Cimpanelli A.M. Grandi • A. Venco

Department of Clinical Medicine

University of Insubria

Ospedale di Circolo

Viale Borri 57,

I-21100 Varese, Italy

e-mail: luigina.guasti@uninsubria.it

C. Scamoni • G. Tomei

Chair of Neurosurgery

University of Insubria, Varese, Italy

S. Sarzi Braga $•$ R. Pedretti

Division of Cardiology

Fondazione Maugeri

Tradate, Varese, Italy

\section{L.T. Mainardi}

Department of Biomedical Engineering

Polytechnic University, Milan, Italy signs. The chest was clear to auscultation, and heart sounds were normal without murmurs. The abdomen was plain and not tender, without masses or organomegalies.

Results of urinalysis, complete blood count, serum electrolyte levels, hepatic-function and renal-function tests showed no abnormalities.

A 24-h ambulatory blood pressure monitoring was then performed (Spacelabs 90207-30, with recording of blood pressure every $15 \mathrm{~min}$ ), revealing a normotensive state throughout the registration $(24 \mathrm{~h}$ mean blood pressure: $125 \pm 12 / 68 \pm 8 \mathrm{mmHg}$; preserved nocturnal blood pressure fall). An echocardiogram revealed normal parietal thickness and function and a radiograph of the chest showed no abnormalities.

\section{Discussion for differential diagnosis}

Prof. Luigina Guasti, Prof. Anna Maria Grandi, Dr. Cinzia Simoni, Dr. Mariagrazia Cimpanelli, Dr. Chiara Crespi: The patient's history was suggestive of secondary forms of hypertension, so we performed the following examinations, with results within the normal range: urinary catecholamines and vanillymandelic acid, 24-h urine-free cortisol, plasma renin activity and aldosterone with their ratio, plasma thyroid hormones. As the results were within the normal ranges, and the clinical presentation was particularly consistent with a preliminary diagnosis of pheochromocytomas, we repeated the urine collection for catecholamines, with normal results. Moreover, we evaluated plasma 5-hydroxyindoleacetic acid in the hypothesis of the presence of a carcinoid tumour. In addition, plasma IGF-1 was determined to exclude excessive GH production.

One month later, the patient experienced one episode of loss of consciousness, immediately after getting out of bed during the night, with a consequent fall and fracture of her wrist. The blood pressure measured immediately after the 
acute episode was $90 / 60 \mathrm{mmHg}$. One week before this episode, she had started to have difficulty swallowing food and drink and mild dysphonia had developed.

During the subsequent six-month period, the patient reported another five hypertensive crises. During one of them she was evaluated in the emergency department of our hospital.

She was anxious, presented with profound malaise and profuse sweating, and her blood pressure was 260/140 $\mathrm{mmHg}$, with the pulse 60 beats/min. Neurologic examination did not reveal pathologic findings. The remainder of the clinical evaluation was normal. Nevertheless, a brain CT scan was performed in the emergency ward, showing normal results.

Because of the suspicion of a renal or cortico-adrenal gland disease, determining the clinical presentation, we ordered an abdominal ultrasonography with Doppler examination of renal arteries, and, to further exclude the presence of a catecholamine-releasing tumour, which remained the strongest clinical suggestion despite the previous negative results of urinary catecholamines, repeated urine collections for catecholamines (also during the day of the crisis) and ${ }^{131}$ I-meta-iodobenzylguanidine scintiscan were performed, without pathological results.

We decided to start an anti-hypertensive treatment with a low dose of carvedilol, which the patient tolerated well.

\section{Further investigations and preliminary findings}

Prof. Achille Venco, Prof. Luigina Guasti, Dr. Cinzia Simoni, Prof. Carlo Scamoni, Prof. Giustino Tomei: On the occasion of a more severe crisis occurring a few weeks later, we admitted the patient to hospital for observation.

The clinical pattern of hypertensive spells and hypotensive episodes suggested a disorder of the regions related to vasomotor modulation. As the CT scan (already performed on our patient) is not the gold standard for the study of the posterior fossa and brain stem, the patient was submitted to a basal and gadolinium MRI of the brain. The sequences revealed an extra-assial mass (diameter $1 \mathrm{~cm}$ ), contrastenhanced, with a nodular morphology, localised in the inferior portions of the cisterna of the right ponto-cerebellar angulus (Fig. 1). Thus the patient was submitted to neurosurgery (right sub-occipital retro-mastoid craniectomy with partial removal of mixed cranial nerve neuroma). The tumour was found to involve the glossopharyngeal nerve. A histopathological diagnosis of Schwannoma was determined.

During the month after surgery the baseline blood pressure (treatment with carvedilol $25 \mathrm{mg}$ and furosemide 25 $\mathrm{mg}$ /day) was normal; however the patient experienced 2 further crises of general malaise associated with high blood pressure levels. The swallowing impairment and disruption in talking worsened after surgery.

\section{Presumptive diagnosis and subsequent tests}

Prof. Achille Venco, Prof. Luigina Guasti, Dr. Simona Sarzi Braga, Dr. Roberto Pedretti: On the basis of both the history and the recent finding of anatomic involvement of the baroreflex arc, Prof. Venco and Prof. Guasti made a presumptive diagnosis of "baroreflex failure" and asked Dr. Pedretti and Dr. Sarzi to confirm the diagnosis. On the basis of the scientific literature on this peculiar form of impairment in baroreflex function, they proposed the pharmacological study of baroreflex sensitivity using the phenylephrine method $[1,2]$.

Phenylephrine $(30 \mu \mathrm{g})$ was injected as an intravenous bolus, followed rapidly by $5 \mathrm{ml}$ of saline with beat-to-beat (RR)-interval and systolic blood pressure continuously monitored. Three injections were performed, with similar

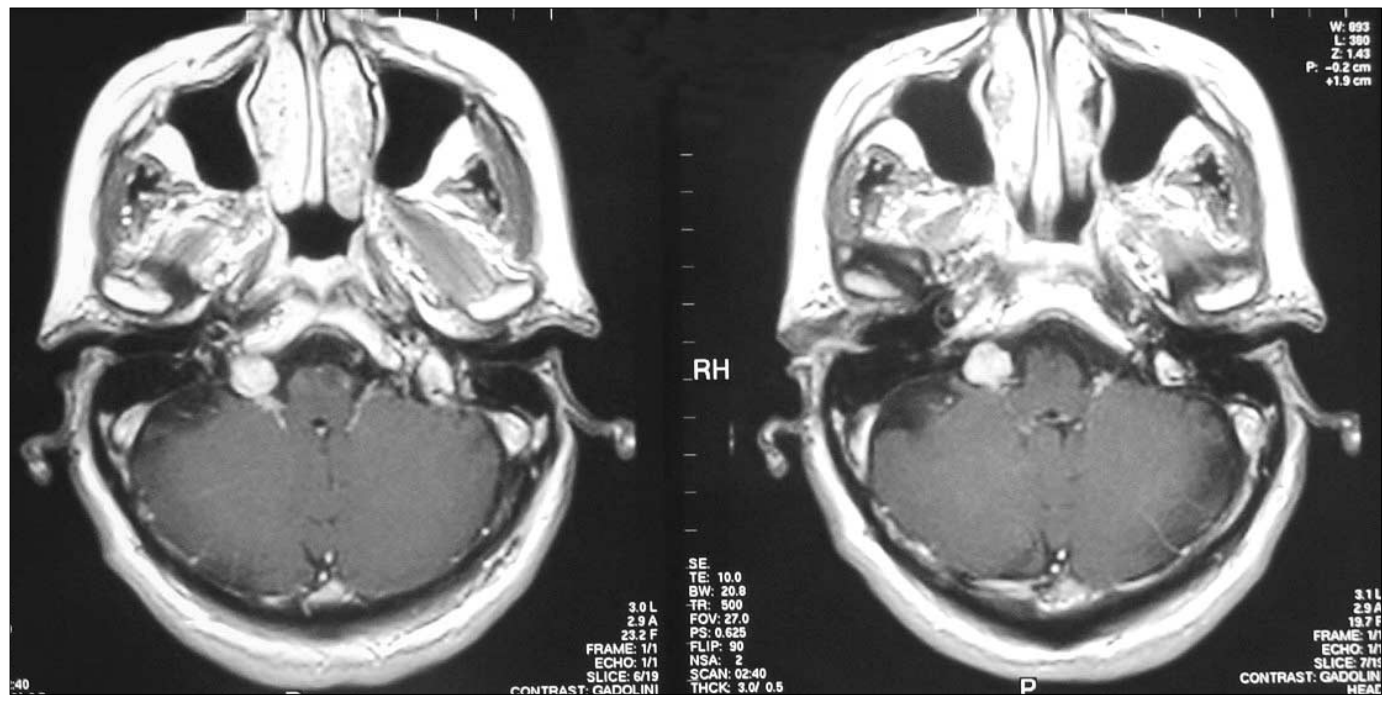

Fig. 1 RMN finding of the lesion localised in the inferior portions of the cisterna of the right ponto-cerebellar angulus 


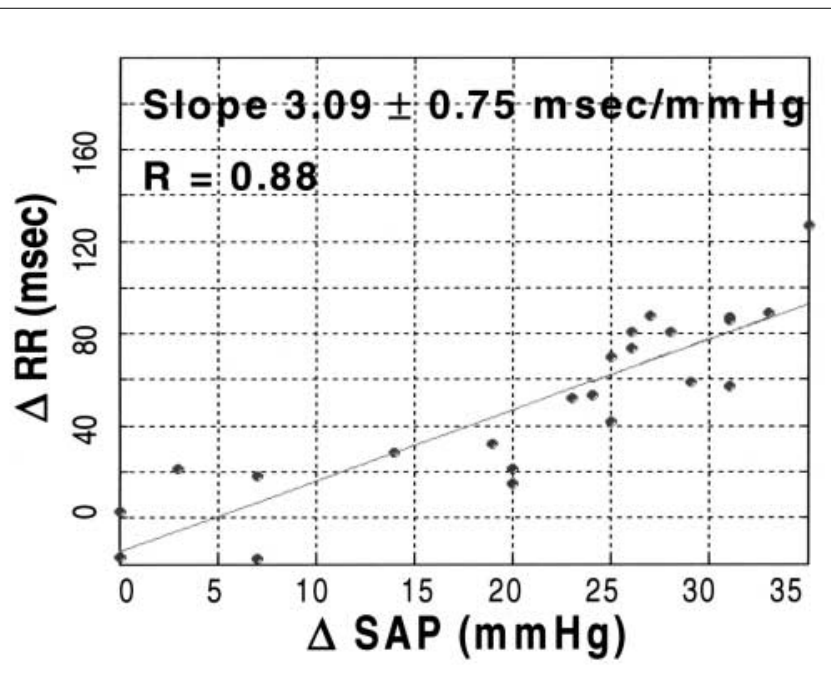

Fig. 2 Relationship between delta systolic arterial pressure (SAP) and delta RR-interval during the phenylephrine tests

results. Figure 2 shows the baroreflex slope. The test showed an important increase in maximal systolic blood pressure (max: $35 \mathrm{mmHg}$ ) in the absence of a significant $\mathrm{RR}$ increase. Moreover, following the data reported in the literature by Robertson et al. [3], limiting the analysis to a $25-\mathrm{mmHg}$ increase we found a decrease in heart rate of 3.5 beats/min for that blood pressure variation.

\section{Diagnosis}

Prof. Achille Venco, Prof. Luigina Guasti: During the first months of the clinical presentation of the case reported here, no anamnestic clue, apart from a nonspecific hypotensive episode, could suggest an involvement of the baroreflex arc in determining the severe hypertensive spells experienced by the patient, which seemed to be more consistent with a diagnosis of pheochromocytoma. Thus the baroreflex impairment preceded the clinical diagnosis of brain tumour mixed cranial nerve neuroma and the neurosurgery by a few months. The results obtained with the phenylephrine method confirmed the preliminary diagnosis of baroreflex failure.

\section{Discussion of diagnosis}

Prof. Achille Venco, Prof. Luigina Guasti, Dr. Simona Sarzi Braga, Dr Luca T. Mainardi: Our diagnosis of baroreflex failure is based on several symptoms (hypertensive crisis associated with tremulousness, irritability, dizziness and profuse sweating, hypotensive episodes, impaired swallowing capacity with cough and disruption in talking) that were clinically documented in our patient and worsened after surgery.

The clinical diagnosis was confirmed by pharmacological baroreflex testing. Several methods have been described for the assessment of vagal reflexes, and among these the bolus injection of vasoactive drugs is the one that has been more extensively used in clinical settings. This method has been in fact used in a large number of patients with different cardiovascular diseases, including myocardial infarction, heart failure and hypertension.

Patients with heart failure show lower slopes for baroreflex sensitivity and older subjects or patients with stable hypertension have a blunted response to phenylephrine. However, our patient showed normal cardiac function and normal/low blood pressure between the hypertensive spells. Moreover, although only a few papers are focused on the study of the pathophysiology of the baroreflex arc in healthy older subjects and uncertainty still remains at least partly due to the different methods used, James et al. [4] reported values significantly higher than that observed in our patient $(7.7 v s .3 \mathrm{~ms} / \mathrm{mmHg})$ in normotensive individuals aged 70 years studied with the Oxford Technique; this finding suggests a marked impairment of the baroreflex gain in our case (of an otherwise healthy 67-year-old woman).

In addition, as Robertson and co-workers [3] expressed reduction of heart rate in beats/min in relation with a 25 $\mathrm{mmHg}$ increase in blood pressure, limiting our analysis to this pressure range, we observed a reduction of 3.5 beats $/ \mathrm{min}$. This value is much lower than those reported both for normal individuals and for patients with stable hypertension and is close to those reported for baroreflex failure.

Baroreflex failure is a relatively rare condition. In the few cases reported in the literature [3, 5-7] the loss of buffering ability is usually secondary to iatrogenic causes such as carotid body tumour resection, neck irradiation, surgical section of glossopharyngeal nerve, carotid by-pass surgery, and unilateral or bilateral carotid endarterectomy [8-12]. Moreover, rare reports describe this syndrome as due to degenerative neurologic disorders involving the brain stem, brain stem stroke or genetic diseases [13-19].

Because of the different possible manifestations of this condition, the differential diagnosis is often difficult [5]. The commonest pattern is that of hypertensive crisis, with profound general malaise, during which the systolic blood pressure could exceed $300 \mathrm{mmHg}$ and is often $>250 \mathrm{mmHg}$ [5]. Another presentation is volatile hypertension. Baseline blood pressure could be normal to elevated but the patient experiences hypertensive spells, lasting minutes to hours, often accompanied by tachycardia. These pressure surges are often elicited by mental or physical stresses. Some patients show an orthostatic tachycardia, defined as an increase in heart rate by more than 30 beats/min from the supine to the upright position. Finally, a rare presentation is 
due to an increased parasympathetic tone with severe bradycardia and asystolia. These patients show hypotension and bradycardia, sometimes leading to a syncope.

\section{Management and follow-up}

Although the few cases in the literature seem to indicate clonidine as an optimal treatment of hypertension associated with baroreflex failure [5], this patient was treated with low doses of carvedilol and furosemide ( 2 drugs that may determine an improvement in baroreflex sensitivity) because these drugs were able to satisfactorily control the symptoms related to the hypertensive spells, which were extremely poorly tolerated by the patient. The crises are now less frequent and the patient and her husband have learned to manage the anxiety that followed her profound malaise and to treat them with short-acting benzodiazepines. Moreover, an increasingly quieter lifestyle has been useful in reducing the spells. The disruption in talking and cough are the main problems at the moment.

\section{Conclusions}

Baroreflex failure is a relatively infrequent/underdiagnosed disease. We propose that examination of the patient should include neuroradiologic evaluation of the ponto-cerebellar angulus when baroreflex failure is clinically suspected or in cases of recurrent hypertensive spells not explained by more frequent clinical disorders.

\section{References}

1. Ecberg DL, Sleight P (1992) Human baroreflex in health and disease. Clarendon Press, Oxford

2. La Rovere MT, Bigger JT, Marcus FI et al for the ATRAMI (Autonomic Tone and Reflexes After Myocardial Infarction) Investigators (1998) Baroreflex sensitivity and heart rate variability in prediction of total cardiac mortality after myocardial infarction. Lancet 351:478-484

3. Robertson D, Hollister AS, Biaggioni I et al (1993) The diagnosis and treatment of baroreflex failure. $\mathrm{N}$ Engl $\mathrm{J}$ Med 329:1449-1455
4. James MA, Robinson TG, Panerai RB, Potter JF (1996) Arterial baroreceptor-cardiac reflex sensitivity in the elderly. Hypertension 28:953-960

5. Ketch T, Biaggioni I, Robertson RM, Robertson D (2002) Four faces of baroreflex failure. Hypertensive crisis, volatile hypertension, orthostatic tachycardia, and malignant vagotonia. Circulation 105:2518-2523

6. Manger WM (1994) Baroreflex failure - a diagnostic challenge. Editorial. N Engl J Med 329:1494-1495

7. Timmers HJLM, Wieling W, Karemaker JM, Lenders JWM (2003) Denervation of carotid baro- and chemoreceptors in humans. J Physiol 553:3-11

8. Timmers HJLM, Karemaker JM, Wieling W et al (2003) Baroreflex and chemoreflex function after bilateral carotid body tumour resection. J Hypertens 21:591-599

9. Timmers HJLM, Karemaker JM, Wieling W et al (2003) Baroreflex control of muscle sympathetic nerve activity after carotid body tumour resection. Hypertension 42:143-149

10. Sharabi Y, Dendi R, Holmes C, Goldstein DS (2003) Baroreflex failure as a late sequela of neck irradiation. Hypertension 42:110-116

11. Biller J, Feinberg WM, Castaldo JE et al (1998) Guidelines for carotid endarterectomy: a statement for healthcare professionals from a special writing group of the stroke council, American Heart Association. Stroke 29:554-562

12. Towne JB, Bernhard VM (1980) The relationship of postoperative hypertension to complications following carotid endarterectomy. Surgery 88:575-580

13. Biaggioni I, Whetsell WO, Jobe J, Nadeau JH (1994) Baroreflex failure in a patient with central nervous system lesions involving the nucleus-tractus-solitarii. Hypertension 23:491-495

14. Diez Porres L, Garcia Iglesias F, Perez Martin G (2003) Multiple paraganglioma: careful with surgery. Rev Clin Esp 203:434-438

15. Hirschowitz BI, Groll A, Ceballos R (1972) Hereditary nerve deafness in 3 sisters with absent gastric motility, small bowel diverticulitis and ulceration and progressive sensory neuropathy. Birth Defects Orig Art Ser 8:27-41

16. Jordan J, Toka HR, Heusser K et al (2000) Severely impaired baroreflex-buffering in patients with monogenic hypertension and neurovascular contact. Circulation 102:2611-2618

17. Milunsky J, DeStefano AL, Huang XL et al (1997) Familiar paragangliomas: linkage to chromosome 11q23 and clinical implications. Am J Med Genet 72:66-70

18. Phillips AM, Jardine DL, Parkin PJ et al (2000) Brain stem stroke causing baroreflex failure and paroxysmal hypertension. Stroke 31:1997-2001

19. Schuster H, Wienker TF, Toka HR et al (1996) Autosomal dominant hypertension and brachydactyly in a Turkish kindred resembles essential hypertension. Hypertension 28:1085-1092 